\title{
Double optical monitoring of dip coating with a time-varying refractive index
}

\author{
Alexandre F. Michels, Thiago Menegotto, Hans Peter H. Grieneisen, Maurício B. Susin, \\ and Flavio Horowitz
}

\begin{abstract}
A brief overview of optical monitoring for vacuum and wet-bench film-deposition processes is presented. Interferometric and polarimetric measurements are combined with regard to simultaneous monitoring of refractive index and physical thickness in real time. Monitoring stability and accuracy are verified during dip coating with a transparent oil standard. This double optical technique is applied to dip coating with a multicomponent zirconyl chloride aqueous solution, whose resulting temporal refractive-index and physical-thickness curves indicate good reproducibility as well as significant sensitivity to changes of film-flow properties during the dip-coating process. (C) 2006 Optical Society of America

OCIS codes: $310.3840,310.6860,260.3160,260.2130$.
\end{abstract}

\section{Introduction}

Interferometric optical monitoring of solid thin films under vacuum deposition has been used for more than half a century for single layers and multilayers, in reflection or transmission, and directly or with witness plates. ${ }^{1,2}$

Application of laser interferometry to monitor spin coating of liquid films, optospinography, started in the last decade and has successfully shown the evolutionary stages of film formation at speeds of as much as 1000 rpm. ${ }^{3-5}$

On the other hand, dip coating is a century-old technique and is still used largely in batch or continuous manufacturing, especially for coating nonplanar or long-dimensional objects. ${ }^{6,7}$ Traditionally, dipcoated film thicknesses are measured by capacitive techniques with typical uncertainties of $\pm 2 \mu \mathrm{m}$, which are also influenced by other parameters, such as substrate- or belt-thickness variations. ${ }^{8}$ Interferometric measurement was reported by Nishida et al. ${ }^{9}$ and, more recently, by Qu et al. ${ }^{10}$ Both studies regard

\footnotetext{
A. F. Michels (michels@if.ufrgs.br), H. P. H. Grieneisen, M. B. Susin, and F. Horowitz are with Instituto de Física, and T. Menegotto and F. Horowitz are with Programa de Pós-Graduação em Microeletrônica (PGMicro), Universidade Federal do Rio Grande do Sul, Campus do Vale, Caixa Postal 15051, 91501-970 Porto Alegre, RS, Brasil.

Received 4 March 2005; accepted 13 August 2005.

0003-6935/06/071491-04\$15.00/0

(C) 2006 Optical Society of America
}

the spatial profile of liquid films at fixed values of time.

Temporal evolution during dip coating was first attained as a result of optical-interference monitoring of Newtonian, nonvolatile mineral oils with a constant refractive index during the dip-coating process. ${ }^{11}$ This allowed the application of a simple model to compare theoretical and experimental results of temporal physical-thickness evolution. ${ }^{12}$ The agreement that was reached made possible contactless monitoring of liquid film-flow properties during the dip-coating process. ${ }^{13}$

However, significant refractive-index variation made explicit interpretation difficult for dip coating with a multicomponent zirconyl chloride aqueous solution. ${ }^{11}$ Although film deposition by dip coating requires little equipment expenses and involves simple experimental procedures, its evolution may involve a complex interplay of physical or chemical mechanisms; this usually leads to a time-varying refractive index during dip coating, which with better monitoring could facilitate greater control and optimization of the process.

A technique for real-time monitoring of optical thin films with varying refractive indices, demonstrated in a vacuum-coating system, was patented by Chou and Arnon ${ }^{14}$ and assigned to Inficon LeyboldHeraeus. This method is based on a wideband spectral measurement at normal incidence and provides refractive indices that are values averaged over the film thickness traversed by the light beam in a manner similarly to that of an earlier spectrophotometry envelope method. ${ }^{15}$ 

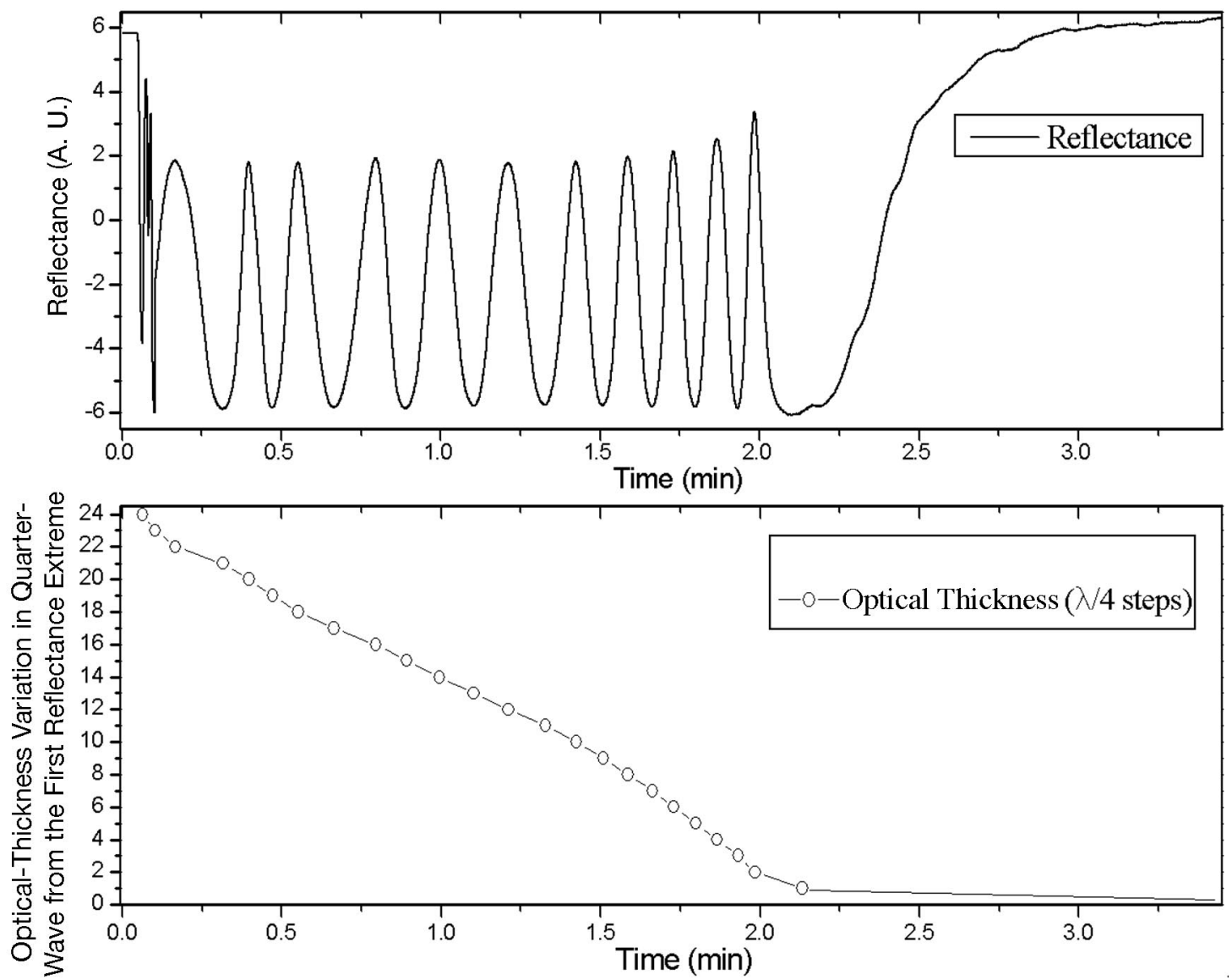

Fig. 1. Temporal evolution of reflectance (top, in arbitrary units) and relative optical thickness (bottom, in quarter-wave steps) for zirconyl solgel at $\lambda=660 \mathrm{~nm}$ during dip coating at a $0.6 \mathrm{~mm} / \mathrm{s}$ withdrawal speed. Measurement realized at $x_{0}=27 \mathrm{~mm}$, with the following process parameters: zirconyl molarity, $0.5 \mathrm{~mol} \mathrm{~L}^{-1}$; relative humidity, $40 \%$; temperature, $22{ }^{\circ} \mathrm{C}$.

In a more recent patent, we introduced a polarimetric method that requires only a singlewavelength laser source and provides the refractive index of a growing or shrinking film at each of its time-varying height positions. ${ }^{16}$ This is particularly convenient for monitoring inhomogeneous coatings during fabrication in real time.

In this study we combine polarimetric and interferometric (double optical) monitoring to determine variable refractive-index and physical-thickness values during dip coating with a complex liquid, as well as to indicate the technique's sensitivity of our method to changes in the film-formation stages during the process.

\section{Experimental Results and Discussion}

In the measurement setup a diode-laser beam with a wavelength $\lambda=660 \mathrm{~nm}$ is measured after reflection by a liquid film flowing on a glass or silicon substrate. As in interferometric optospinography, ${ }^{3,4}$ the reflected light at normal incidence is compared with a reference signal that is later processed by a lock-in amplifier and by a computer with an analog-to-digital converter. ${ }^{12,13}$ In the polarimetric measurement the laser light, polarized in the plane of incidence, impinges onto the sample at a variable angle. ${ }^{16}$
The high acquisition rate, typically of the order of $3.5 \mathrm{kHz}$, and the proper alignment of the optical system, leading to a small illuminated spot on the sample (diameter of less than $0.5 \mathrm{~mm}$ ) at $x=x_{0}$, were carefully maintained for detailed monitoring of the dip-coating process.

The low power of the laser spot did not affect the temporal pattern of the interference fringes, thus indicating that local heating did not influence the film properties. The bath container was routinely kept inside a transparent chamber to prevent contamination as well as temperature inhomogeneities. The sample and the optical system were physically isolated from the dip coater to avoid vibration-induced film fluctuations.

Experiments were performed by using a multicomponent zirconyl chloride aqueous solution at several withdrawal speeds. Interferometric patterns were obtained during dip coating, as illustrated in Fig. 1, where each interval between successive extremes corresponds to an optical-thickness variation of $\lambda / 4$.

Direct measurement of the varying refractive index during the dip-coating process was then achieved by the polarimetric measurement in real time. Its combination with the above-mentioned interferometric determination of the optical-thickness variation also 


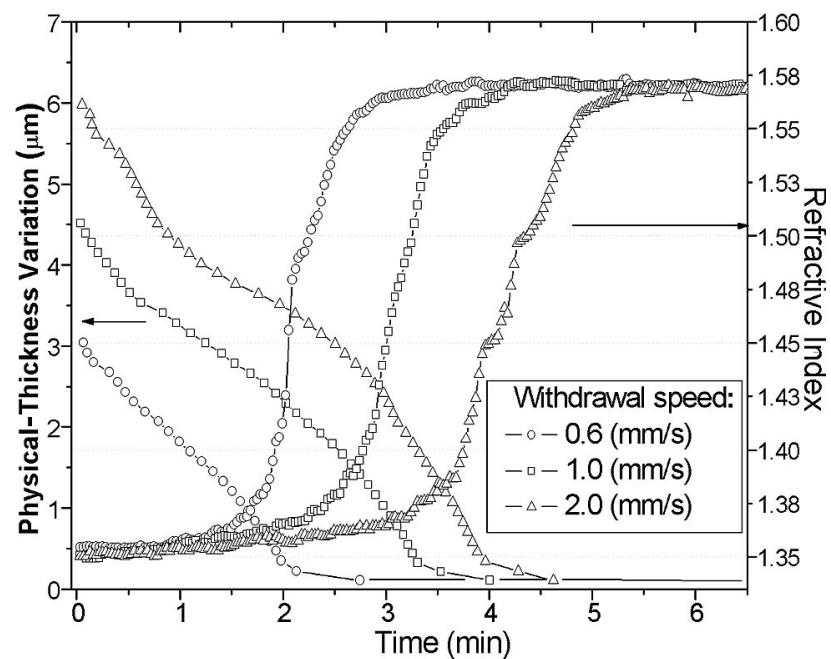

Fig. 2. Temporal evolution of physical thickness and refractive index during dip coating for a multicomponent zirconyl chloride aqueous solution at distinct withdrawal speeds. Measurement realized at $x_{0}=27 \mathrm{~mm}$, with the following process parameters: zirconyl molarity, $0.5 \mathrm{~mol} \mathrm{~L}^{-1}$; relative humidity, $40 \%$; temperature, $22{ }^{\circ} \mathrm{C}$.

provided information about physical-thickness evolution during dip coating, as shown in Fig. 2.

Initially, the stability of the refractive-index monitor was verified by using an oil standard at a fixed temperature, as presented in Fig. 3. This ensured that film properties remained constant throughout the process and provided a comparison with the value obtained by the Abbe refractometry, demonstrating monitor accuracy within experimental uncertainties.

For solgel films, additional process parameters were kept constant to achieve monitoring reproducibility (as indicated in Fig. 4), including the measurement coordinate position at the substrate (see $x_{0}$ in

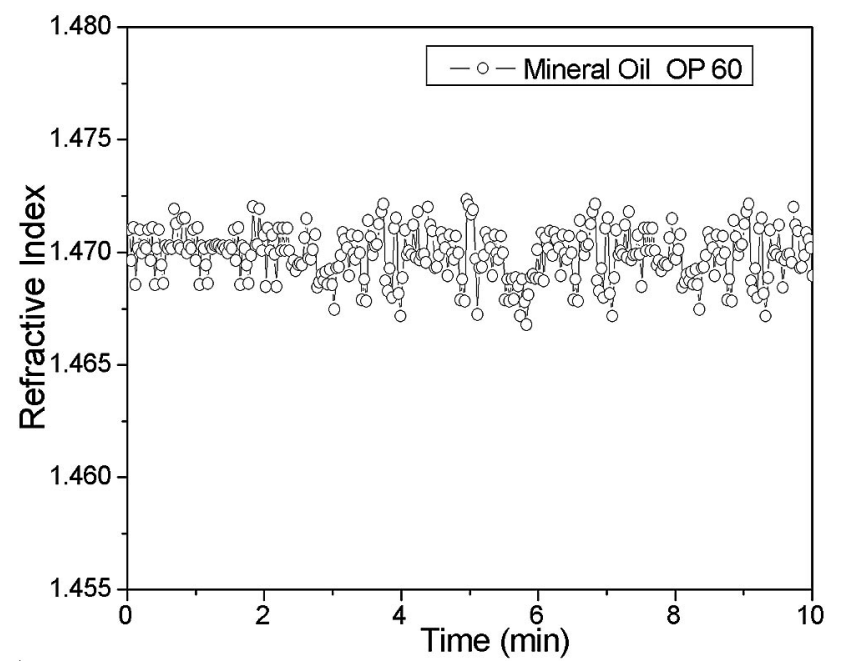

Fig. 3. Stability test of the refractive-index monitor during dip coating at a $2 \mathrm{~mm} / \mathrm{s}$ withdrawal speed, using a Newtonian, nonvolatile oil standard at a constant temperature of $24^{\circ} \mathrm{C}$. The refractive index measured by the Abbe refractometry, also at $660 \mathrm{~nm}$ and $24^{\circ} \mathrm{C}$, was $1.470 \pm 0.001$.

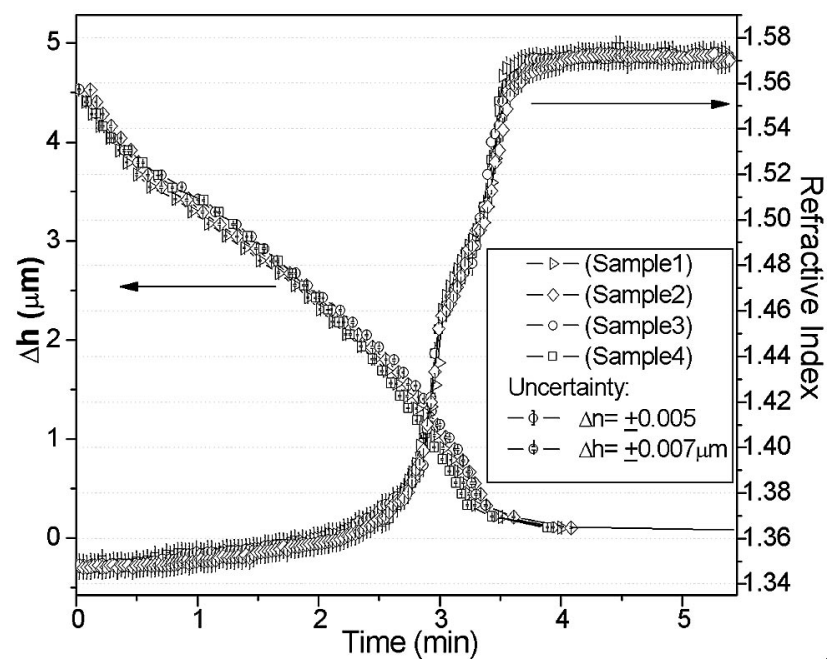

Fig. 4. Reproducibility of the refractive index $(n)$ and the physical-thickness $(h)$ temporal evolution during dip coating at a $1 \mathrm{~mm} / \mathrm{s}$ withdrawal speed, illustrated with four runs with a multicomponent zirconyl chloride aqueous solution. Measurement realized at $x_{0}=27 \mathrm{~mm}$ with the following process parameters: zirconyl molarity, $0.5 \mathrm{~mol} \mathrm{~L}^{-1}$; relative humidity, $40 \%$; temperature, $22{ }^{\circ} \mathrm{C}$.

Fig. 5), the withdrawal speed $(U)$, the substrate surface quality, the temperature, the relative humidity, and the sol composition. ${ }^{17}$

The influence of each of these process parameters in the variation of the physical thickness and the refractive index can be evaluated in real time through the double optical-monitoring method. Consequently, film-formation stages can be identified, as exemplified by different withdrawal speed parameters in Fig. 2.

The zirconyl chloride solgel dip-coating process can be divided into several film-formation stagesdominated by mass loss, solvent evaporation, or consequent structural changes-as clearly indicated by the curve inflections in Fig. 2. This demonstrates significant sensitivity of the double monitoring method to the evolution of film properties.

\section{Concluding Remarks}

Double optical monitoring, which combines interferometric and polarimetric measurements, was applied to dip coating with a complex transparent liquid. The refractive indices and physical thicknesses were determined in real time, with precisions of \pm 0.005 and $\pm 0.007 \mu \mathrm{m}$, respectively. The stability and accuracy of the method were also verified with an oil standard. The resulting reproducibility and sensitivity to flow properties, as shown by the time-varying refractiveindex and physical-thickness curves of a zirconyl solgel under dip coating, suggest that this method can accommodate a more detailed investigation of filmformation stages and that it can be applied to vacuum deposition of inhomogeneous coatings.

This research was partially supported by the Brazilian agencies Conselho Nacional de Desenvolvimento 


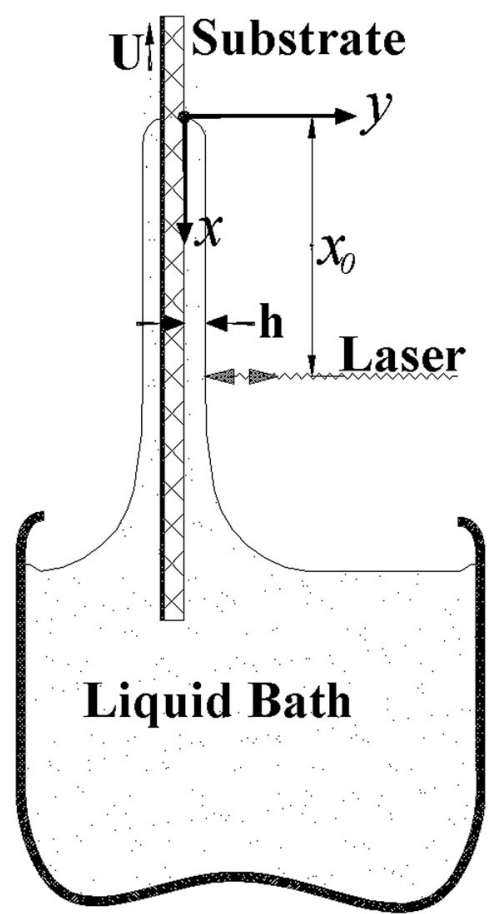

Fig. 5. Illustration of the dip-coating batch process in which laser probing is shown: $U$ is the withdrawal speed and $h$ is the film's physical thickness on the $y$ axis. The illuminated area under the measurement corresponds to a fixed value $x_{0}$ on the vertical $x$ axis.

Científico e Tecnológico (CNPq) and Coordenação de Aperfeiçoamento de Pessoal de Nível Superior (CAPES).

\section{References}

1. H. A. Macleod, Thin-Film Optical Filters, 2nd ed. (Macmillan, 1986).

2. K. H. Bernhdt, "Film thickness and deposition rate monitoring devices and techniques of production films of uniform thickness," in Physics of Thin Films, 6th ed., D. E. Hass and R. E. Thun, eds. (Academic, 1966), Vol. 3, pp. 1-59.

3. F. Horowitz, E. M. Yeatman, E. Dawnay, and A. Fardad, "Optics as a key to spin coating sol-gel films," in Optics as a Key to
High Technology, G. Akos, T. Lippenyi, G. Lupkovics, and A. Podmaniczky, eds., Proc. SPIE 1983, 1022-1023 (1993).

4. F. Horowitz, E. M. Yeatman, E. Dawnay, A. Fardad, and M. Green, "Real-time optical monitoring of spin coating," J. Phys. III 3, 2059-2063 (1993).

5. D. E. Haas, J. N. Quijada, S. J. Picone, and D. P. Birnie, "Effect of solvent evaporation rate on skin formation during spin coating of complex solutions," in Sol-Gel Optics V, B. Dunn, E. Pope, H. K. Schmidt, and M. Yamane, eds., Proc. SPIE 3943, 280-284 (2000)

6. L. E. Scriven, "Physics and applications of dip coating and spin coating," Mater. Res. Soc. 121, 717-729 (1988).

7. P. R. Schunk, A. J. Hurd, and C. J. Brinker, "Free-meniscus coating processes," in Liquid Film Coating, S. F. Kistler and P. M. Schweizer, eds. (Chapman \& Hall, 1997), pp. 673-708.

8. R. P. Spiers, C. V. Subabaraman, and W. L. Wilkinson, "Free coating of a Newtonian liquid onto a vertical surface," Chem. Eng. Sci. 29, 389-396 (1973).

9. F. Nishida, J. M. McKiernan, B. Dunn, J. I. Zink, C. J. Brinker, and A. J. Hurd, "In situ fluorescence probing of the chemical changes during sol-gel thin film formation," J. Am. Ceram. Soc. 78, 1640-1648 (1995).

10. D. Qu, E. Rancé, and S. Garoff, "Dip coated films of volatile liquids," Phys. Fluids 14, 1154-1165 (2002).

11. F. Horowitz, A. F. Michels, P. Alcantara Jr., M. B. Pereira, A. P. Rizzato, and C. V. Santilli, "Real-time interferometric monitoring of dip coating," in Optics for the Next Millenium, A. J. Glass, J. W. Goodman, M. Chang, A. H. Guenter, and T. Asakura, eds., Proc. SPIE 3749, 729-730 (1999).

12. A. F. Michels, T. Menegotto, and F. Horowitz, "Interferometric monitoring of dip coating," Appl. Opt. 43, 820-823 (2004).

13. F. Michels, T. Menegotto, and F. Horowitz, "Optically monitored dip coating as a contactless viscometry method for liquid films," Appl. Opt. 44, 912-915 (2005).

14. J. T. Chou and O. Arnon, "Refractive index measurement of optical thin-film," U.S. patent 4,335,961 (22 June 1982).

15. J. C. Manifacier, J. Gasiot, and J. P. Fillard, "A simple method for the determination of the optical constants $n, k$ and thickness of a weakly absorbing thin film," J. Phys. E 9, 1002-1004 (1976).

16. F. Horowitz, A. F. Michels, H. P. Grieneisen, and J. A. Lisboa, "Aparelho Monitorador para Medida Direta e Multi-Angular do Índice de Refração, Método e Uso do Mesmo," Brazil patent 0305389-0 (28 June 2005).

17. L. A. Chiavacci, S. H. Pulcinelli, and C. V. Santilli, "Structural and phenomenological characterization of the thermoreversible sol-gel transition of a zirconyl aqueous precursor modified by sulfuric acid," Chem. Mater. 10, 986-993 (1998). 